\title{
Using Spatio-Temporal Information in API Calls with Machine Learning Algorithms for Malware Detection
}

\author{
Faraz Ahmed, Haider Hameed, M. Zubair Shafiq, Muddassar Farooq \\ Next Generation Intelligent Networks Research Center (nexGIN RC) \\ National University of Computer and Emerging Science (FAST-NUCES) \\ Islamabad 44000, Pakistan \\ \{faraz.ahmed,haider.hameed,zubair.shafiq,muddassar.farooq\}@nexginrc.org
}

\begin{abstract}
Run-time monitoring of program execution behavior is widely used to discriminate between benign and malicious processes running on an end-host. Towards this end, most of the existing run-time intrusion or malware detection techniques utilize information available in Windows Application Programming Interface (API) call arguments or sequences. In comparison, the key novelty of our proposed tool is the use of statistical features which are extracted from both spatial (arguments) and temporal (sequences) information available in Windows API calls. We provide this composite feature set as an input to standard machine learning algorithms to raise the final alarm. The results of our experiments show that the concurrent analysis of spatio-temporal features improves the detection accuracy of all classifiers. We also perform the scalability analysis to identify a minimal subset of API categories to be monitored whilst maintaining high detection accuracy.
\end{abstract}

\section{Categories and Subject Descriptors}

D.4.6 [Security and Protection]: Invasive software (e.g., viruses, worms, Trojan horses); I.5 [Computing Methodologies]: Pattern Recognition

\section{General Terms}

Algorithms, Experimentation, Security

\section{Keywords}

API calls, Machine learning algorithms, Malware detection, Markov chain

\section{INTRODUCTION}

The API calls facilitate user mode processes to request a number of services from the kernel of Microsoft Windows operating system. A program's execution flow is essentially

Permission to make digital or hard copies of all or part of this work for personal or classroom use is granted without fee provided that copies are not made or distributed for profit or commercial advantage and that copies bear this notice and the full citation on the first page. To copy otherwise, to republish, to post on servers or to redistribute to lists, requires prior specific permission and/or a fee.

AISec'09, November 9, 2009, Chicago, Illinois, USA.

Copyright 2009 ACM 978-1-60558-781-3/09/11 ...\$10.00. equivalent to the stream of API calls [2]. Moreover, the Microsoft Windows provides a variety of $\mathrm{API}^{1}$ calls of different functional categories - registry, memory management, sockets, etc. Every API call has a unique name, set of arguments and return value. The number and type of arguments may vary for different API calls. Likewise, the type of return value may also differ.

In the past, a significant amount of research has been focused on leveraging information available in API calls for monitoring a program's behavior. The behavior of a program can potentially highlight anomalous and malicious activities. The seminal work of Forrest et al. in [9] leveraged temporal information (fixed length sequences of system calls) to discriminate between benign and malicious Unix processes [16]. Later, Wepsi et al. proposed an improved version with variable length system call sequences [17]. In a recent work, the authors propose to extract semantics by annotating call sequences for malware detection [6]. The use of flow graphs to model temporal information of system calls has been proposed in [5]. An important shortcoming of these schemes is that a crafty attacker can manipulate temporal information to circumvent detection [12], [15].

Subsequently, Mutz et al. proposed a technique that utilizes information present in system call arguments and return values [11]. The results of their experiments reveal that using spatial information enhances the robustness of their proposed approach to mimicry attacks and other evasion attempts. Another technique, M-LERAD, uses simple white-lists of fixed-length sequences and system call argument values for anomaly detection [14]. The authors report that the accuracy of the composite scheme is better than either of the standalone spatial and temporal approaches.

In this paper, we propose a composite malware detection scheme that extracts statistical features from both spatial and temporal information available in run-time API calls of Windows operating systems. The thesis of our approach is that leveraging spatio-temporal information in run-time API calls with machine learning algorithms can significantly enhance the malware detection accuracy. Figure 1 provides the architecture of our proposed scheme. Our tool consists of two modules: (1) offline training module applies machine learning algorithms on the available data to develop a training model, and (2) online detection module extracts spatiotemporal features at run-time and compares them with the

\footnotetext{
${ }^{1}$ The latest Microsoft Windows operating systems provide both Native API (system calls) and Windows API. Throughout this paper, by API we refer to both of them unless stated otherwise.
} 


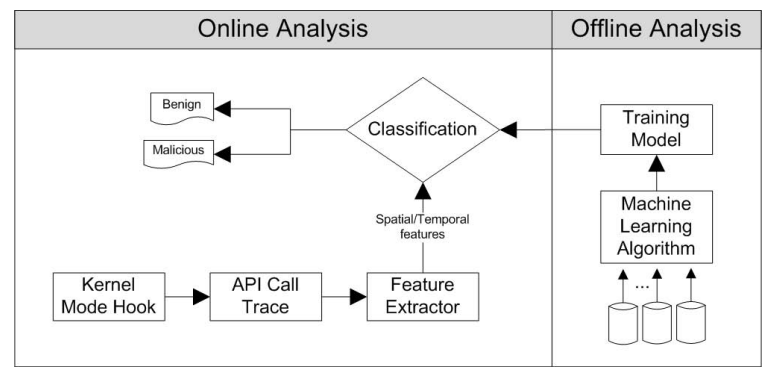

Figure 1: Block diagram of our proposed malware detection tool

training model to classify a running process as benign or malware.

In our scheme, spatial features are generally statistical properties such as means, variances and entropies of address pointers and size parameters. The temporal information is modeled using $n^{t h}$ order discrete time Markov chain with $k$ states, where each state corresponds to a particular API call. In the training phase, two separate Markov chains are constructed for benign and malicious traces that result in $k^{n}$ different transition probabilities for each chain. The less 'discriminative' transitions are pruned by using an informationtheoretic measure called information gain $(I G) . \Gamma$ transitions with the highest values of $I G$ are selected as boolean features. The extracted spatial and temporal features are then given as input to the standard machine learning algorithms for classification.

We have used a commercial API call tracer that uses the kernel-mode hook to record the logs for running processes on Microsoft Windows XP. To reduce the complexity of runtime tracing, we have short-listed 237 core API calls from six different functional categories such as socket, memory management, processes, and threads etc. We have collected system call logs of 100 benign programs. Moreover, we have collected system call traces of 117 trojans, 165 viruses and 134 worms. These malware are obtained from a publicly available collection called 'VX Heavens Virus Collection' [3]. The results of our experiments show that our system provides an accuracy of 0.98 on the average. We have also carried out the scalability analysis to identify a minimal subset of API categories to be monitored whilst maintaining high detection accuracy. The results of the scalability analysis show that monitoring only memory management and file $I / O$ API calls can provide an accuracy of 0.97 .

The rest of the paper is organized as follows. In the next section we provide an introduction to the Windows API. In Section 3, we explore different design dimensions to extract information - spatial and temporal - available in the Windows API calls. In Section 4, we present the mathematical formulation of our problem and then use it to model and quantify spatio-temporal features. In Section 5, we present details of our dataset, experimental setup and discuss the results of experiments. Section 6 concludes the paper with an outlook to our future research. We provide brief details of machine learning classifiers in the accompanying technical report [4].
Table 1: Categorization of API calls used in this study

\begin{tabular}{|c||c|}
\hline API Category & Explanation \\
\hline \hline Registry & registry manipulation \\
\hline Network Management & manage network related operations \\
\hline Memory Management & manage memory related functionalities \\
\hline File Input/Output (I/O) & operations like reading from disk \\
\hline Socket & socket related operations \\
\hline Processes \& Threads & manage processes and thread \\
\hline Dynamic-Link Libraries & manipulations of DLLs \\
\hline
\end{tabular}

\section{WINDOWS APPLICATION PROGRAM- MING INTERFACE (API)}

Microsoft provides its Windows application developers with the standard API enabling them to carry out an easy and rapid development process. The Windows API provides all basic functionalities required to develop a program. Therefore, a developer does not need to write the codes for basic functionalities from scratch. One of the reasons for popularity of Windows operating system among developers is its professionally documented and diverse Windows API. An application developer can simply call the appropriate functions in the Windows API to create rich and interactive environments such as graphical user interfaces. Due to their widespread usage, the functionality of all Windows applications depends on the Windows API [2]. Therefore, a Windows application can be conceptually mapped to a stream of Windows API calls. Monitoring the call stream can effectively provide insights into the behavior of an application. For example, a program calling WriteFile is attempting to write a file on to the hard disk and a program calling RegOpenKey is in fact trying to access some key in the Windows registry. The Windows API provides thousands of distinct API calls serving diverse functionalities. However, we have short-listed 237 API calls which are widely used by both benign Windows applications and malware programs. We refer to the short-listed calls as the core-functionality API calls.

We further divide the core-functionality API calls into seven different categories: (1) registry, (2) network management, (3) memory management, (4) file I/O, (5) socket, (6) processor and threads, and (7) dynamically linked libraries (DLLs). A brief description of every category is provided in Table 1. The majority of benign and malware programs use API calls from one or more of the above-mentioned categories. For example, a well-known Bagle malware creates registry entries uid and frun in HKEY_CURRENT_USER $\backslash$ SOFTWARE $\backslash$ Windows. It also collects the email addresses from a victim's computer by searching files with the extensions .wab, .txt, .htm, .html. Moreover, Bagle opens a socket for communicating over the network.

\section{MONITORING PROGRAM BEHAVIOR USING WINDOWS API CALLS}

The API calls of different programs have specific patterns which can be used to uniquely characterize their behavior. In this section, we report comparative analysis of the behavior of benign and malware programs. The objective of the study is to build better insights about the data model used by our malware detection scheme. We will explore design 
options for our scheme along two dimensions: (1) local vs. global, and (2) spatial vs. temporal.

\subsection{Dimension 1: Local/Global Information}

The execution of a program may result in a lengthy trace of API calls with several thousand core-functionality entries. The information can either be extracted locally (considering individual API calls) or globally (considering API trace as a whole). The feature extraction process done locally has a number of advantages: (1) the extracted information is specific to an instance of a particular type of an API call, and (2) a large number of features can be extracted. However, it is well-known that locally extracted features are vulnerable to basic evasion attempts such as obfuscation by garbage call insertion [15], [12]. In comparison, the globally extracted features are more robust to basic evasion attempts. Therefore, we extract global information by taking into account complete API call streams rather than the individual calls.

\subsection{Dimension 2: Spatial/Temporal Informa- tion}

Recall that every API call has a unique name, a set of arguments and a return value. Moreover, different API calls may have different number (or type) of arguments and different type of return value. We can extract two types of information from a given stream of API calls: (1) spatial information (from arguments and return values of calls), and (2) temporal information (from sequence of calls). We first build intuitive models of spatial and temporal information, which are followed by formal models.

\subsubsection{Spatial Information.}

All API functions have a predefined set of arguments. Let us discuss the type and the number of arguments. The arguments of API calls are generally address pointers or variables that hold crisp numerical values. In [14], the authors have used white-lists (containing valid values) for a particular argument of a given API call. However, using the actual values of arguments to design features is not a good idea given the ease of their manipulation. To this end, we propose to use statistical characteristics of same arguments of an API call across its different invocations. We use statistical and information theoretic measures - such as mean, variance, entropy, minimum, and maximum values of the pointers that are passed as arguments in API calls.

Table 2 provides examples of the spatial information that can be extracted from API call arguments. LocalAlloc function belongs to memory management API and has two input arguments: uFlags and uBytes. uBytes argument determines the number of bytes that need to be allocated from the heap memory. It is evident from Table 2 that LocalAllocuBytesMean - mean of uBytes parameter of LocalAlloc call - has relatively low values for benign programs compared with different types of malware. hMem parameter of GlobalFree function has significantly large variance for benign programs as compared to all types of malware.

\subsubsection{Temporal Information.}

Temporal information represents the order of invoked API calls. The majority of researchers have focused on using the temporal information present in the stream of API calls. Call sequences and control flow graphs are the most popular techniques [9], [5]. It has been shown that certain call se- quences are typical representations of benign programs. For example, Table 2 and Figure 2 contain two sequences which occur frequently in API call traces of benign programs and are absent from API call traces of malware software. These API calls are related to the memory management functionality. We have observed that benign programs - mostly for graphics-related activities - extensively utilize memory management functionalities. Intuitively speaking, malware writers suffice on lean and mean implementations of malware programs with low memory usage so that they can remain unnoticed for as long as possible.

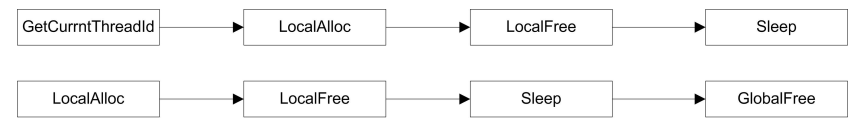

Figure 2: API call sequences present in benign traces and absent in malware traces

Similarly, it is shown that certain call sequences form signatures of malware software. Figure 3 shows two API call sequences which frequently occur in execution traces of malware but are absent from the traces of a benign program. The API functions in these sequences are generally used to access the Windows registry - a gateway to the operating system. A malware is expected to use these API calls to access and alter information in the registry file. Two sequences shown in Figure 3 represent a program which first makes a query about a registry entry and then sets it with a proper value. The second sequence opens a registry key, retrieves data in the key and then alters it.

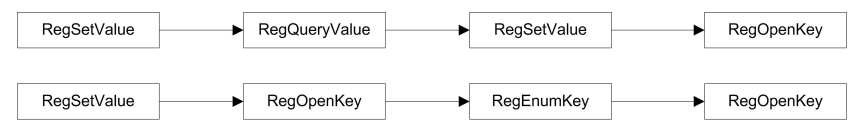

Figure 3: API call sequences present in malware traces and absent in benign traces

Having developed an intuitive model of our data we will now focus on the formal modeling of spatial and temporal information.

\section{MODELING AND QUANTIFICATION OF SPATIO-TEMPORAL INFORMATION}

We have already explained in Section 3 that we can extract two types of information - spatial and temporal - from API calls. In this section, we present formal models to systematically quantify these features. We start by presenting a formal definition of API calls that will help in better understanding of the formal model for spatio-temporal information.

\subsection{Formal Definitions}

An API call $i$ can be formally represented by a tuple $\left(T_{i}\right)$ of the form:

$$
\left(S_{i}, F_{(i, 1)}, F_{(i, 2)}, \ldots, F_{(i, \hbar(i))}, R_{i}\right),
$$

where $S_{i}$ is its identity (string name), $R_{i}$ is its return value, and $\hbar(i)$ is its number of arguments. The range of $\hbar(i)$ in our study is given by $R(\hbar)=\{0,1,2,3,4,5\}$.

The Windows operating system and third-parties provide a large set $\mathbb{S}_{T}$ of API calls. Each call is represented by 
Table 2: Examples of information extracted from API calls

\begin{tabular}{|c||c|c|c||c|c|c|}
\hline \multicolumn{1}{|c||}{} & \multicolumn{3}{c||}{ Benign } & \multicolumn{3}{c|}{ Malware } \\
\hline Information & Installations & Network Utilities & Misc. & Trojans & Viruses & Worms \\
\hline \hline LocalAllocuBytesMean & 48.80 & 18.33 & 59.72 & 95.95 & 111.02 & 100.41 \\
\hline GlobalFreehMemVar & 261.08 & 298.77 & 274.56 & 58.13 & 49.63 & 51.46 \\
\hline Seq-1 & 33.33 & 66.67 & 47.83 & 0.00 & 0.00 & 0.00 \\
\hline Seq-2 & 33.33 & 66.67 & 44.93 & 0.00 & 0.00 & 0.00 \\
\hline
\end{tabular}

a unique string $S_{i} \in \mathbb{S}_{T}$. In our study, we only consider a core-functionality subset $\mathbb{S} \subset \mathbb{S}_{T}$. $\mathbb{S}$ is further divided into seven functional subsets: (1) socket $\mathbb{S}_{\text {sock }}$, (2) memory management $\mathbb{S}_{m m},(3)$ processes and threads $\mathbb{S}_{\text {proc }},(4)$ file I/O $\mathbb{S}_{i o}$, (5) dynamic linked libraries $\mathbb{S}_{d l l}$, (6) registry $\mathbb{S}_{r e g}$, and (7) network management $\mathbb{S}_{n m}$. That is, $\mathbb{S} \supseteq$ $\left(\mathbb{S}_{\text {sock }} \bigcup \mathbb{S}_{m m} \bigcup \mathbb{S}_{\text {proc }} \bigcup \mathbb{S}_{i o} \bigcup \mathbb{S}_{\text {dll }} \bigcup \mathbb{S}_{r e g} \bigcup \mathbb{S}_{n m}\right)$

A trace of API calls (represented by $\Theta_{P}$ ) is retrieved by executing a given program $(P)$. The trace length $\left(\left|\Theta_{P}\right|\right)$ is an integer variable. $\Theta_{P}$ is an ordered set which is represented as:

$$
<T_{(p, 1)}, T_{(p, 2)}, \ldots, T_{\left(p,\left|\Theta_{P}\right|\right)}>
$$

It is important to note that executing a given program $P$ on different machines with different hardware and software configurations may result in slightly different traces. In this study, we assume that such differences, if any, are practically negligible. We are now ready to explore the design of spatial and temporal features on the basis of above-mentioned formal representations of API calls.

\subsection{Spatial Features}

API calls have input arguments and return values which can be utilized to extract useful features. Recall that the majority of these arguments are generally address pointers and size parameters. We now present the modeling process of spatial information.

\subsubsection{Modeling Spatial Information.}

The address pointers and size parameters can be analyzed to provide a valuable insight about the memory access patterns of an executing program. To this end, we have handpicked arguments from a number of API calls such as CreateThread, GetFileSize, SetFilePointer, etc. The selected fields of API call arguments, depending on the category of API call, can reflect the behavior of a program in terms of network communication $\left(\mathbb{S}_{\text {sock }}\right)$, memory access $\left(\mathbb{S}_{m m}\right)$, process $/$ thread execution $\left(\mathbb{S}_{\text {proc }}\right)$ and file I/O $\left(\mathbb{S}_{i o}\right)$. We now choose appropriate measures to quantify the selected spatial information.

\subsubsection{Quantification.}

We use fundamental statistical and information theoretic measures to quantify the spatial information. These measures include mean $(\mu)$, variance $\left(\sigma^{2}\right)$, entropy $(H)$, minimum $(\min )$, and maximum $(\max )$ of given values. Mathematically, for $F_{(i, j)} \in \Delta_{F_{(i, j)}}$, we define the statistical properties mentioned above for $j^{t h}$ argument of an $i^{t h}$ API call as:

$$
\mu=E\left\{F_{(i, j)}\right\}=\sum_{a_{k} \in \Delta_{F_{(i, j)}}}\left(a_{k} \cdot \operatorname{Pr}\left\{F_{(i, j)}=a_{k}\right\}\right)
$$

$$
\begin{gathered}
\sigma^{2}=\operatorname{var}\left\{F_{(i, j)}\right\}=\sum_{a_{k} \in \Delta_{F_{(i, j)}}}\left(\left(a_{k}-E\left\{F_{(i, j)}\right\}\right)^{2} \cdot \operatorname{Pr}\left\{F_{(i, j)}=a_{k}\right\}\right) \\
H\left\{F_{(i, j)}\right\}=-\sum_{a_{k} \in \Delta_{F_{(i, j)}}} a_{k} \log _{2}\left(a_{k}\right) \\
\min \left\{F_{(i, j)}\right\}=a_{k}^{*} \mid a_{k}^{*} \leq a_{k}, \quad \forall a_{k} \in \Delta_{F_{(i, j)}} \\
\max \left\{F_{(i, j)}\right\}=a_{k}^{*} \mid a_{k}^{*} \geq a_{k}, \quad \forall a_{k} \in \Delta_{F_{(i, j)}}
\end{gathered}
$$

\subsection{Temporal Features}

Given a trace (represented by $\Theta_{P}$ ) for the program $P$, we can treat every call $S_{i}$ as an element of $S_{p}=<S_{0}, S_{1}, \ldots$, $S_{\left|\Theta_{P}\right|}>$, where $S_{p}$ represents a sequence of API calls. Without the loss of generality, we can also treat every consecutive $n$ calls in $S_{p}$ as an element. For example, if $S_{p}=$ $\langle a, b, c, d, e\rangle$, and we consider two consecutive calls as an element $(n=2)$, we get the sequence as $\langle a b, b c, c d, d e\rangle$. This up-scaling also increases the dimensionality of the distribution from $k$ to $k^{n}$. This process not only increases the underlying information but may also result in sparse distributions because of lack of training data. Therefore, an inherent tradeoff exists between the amount of information, characterized by entropy, and the minimum training data required to build a model. Consequently, selecting appropriate value of $n$ is not a trivial task and several techniques are proposed to determine its appropriate value.

It is important to note that the up-scaled sequence with $n=2$ is in fact a simple joint distribution of two sequences with $n=1$, and so on. The joint distribution may contain some redundant information which is relevant for a given problem. Therefore, we need to remove the redundancy for accurate analysis. To this end, we have analyzed a number of statistical properties of the call sequences. A relevant property that has provided us interesting insights into the statistical characteristics of call sequences is the correlation in call sequences [13].

\subsubsection{Correlation in Call Sequences.}

Autocorrelation is an important statistic for determining the order of a sequence of states. Autocorrelation describes the correlation between the random variables in a stochastic process at different points in time or space. For a given lag $t$, the autocorrelation function of a stochastic process, $X_{m}$ (where $m$ is the time/space index), is defined as:

$$
\rho[t]=\frac{E\left\{X_{0} X_{t}\right\}-E\left\{X_{0}\right\} E\left\{X_{t}\right\}}{\sigma_{X_{0}} \sigma_{X_{t}}},
$$

where $E\{$.$\} represents the expectation operation and \sigma_{X_{m}}$ is the standard deviation of the random variable at time/space 


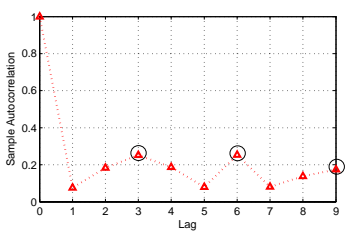

(a) Matlab

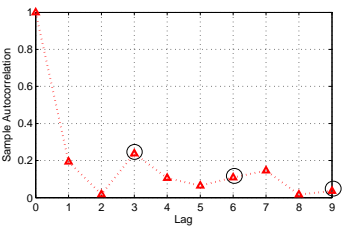

(c)

Virus.Win32.Flechal

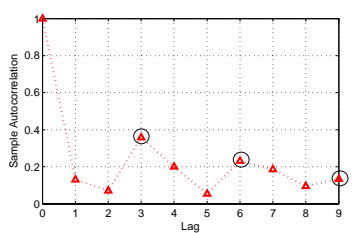

(b) Trojan.Win32.Aimbotee

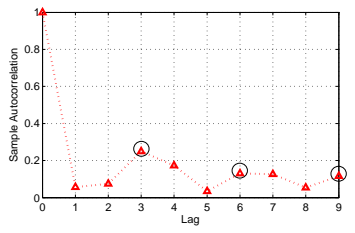

(d) Worm.Win32.ZWQQ.a
Figure 4: Sample autocorrelation functions of API call sequences show peaks at $n=3,6$, and 9 for benign, trojan, virus, and worm executables.

lag $m$. The value of the autocorrelation function lies in the range $[-1,1]$, where $\rho[t]=1$ means perfect correlation at lag $t$ (which is obviously true for $n=0$ ), and $\rho[t]=0$ means no correlation at all at lag $t$.

To observe the dependence level in call sequences $S_{p}$, we calculate sample autocorrelation functions for benign and malware programs. Figure 4 shows the sample autocorrelation functions plotted versus the lag. It is evident that call sequences show a $3^{\text {rd }}$ order dependence because the autocorrelation shows peaks at $n=3,6,9, \ldots$ in most of the cases. This fact will be useful as we develop our statistical model of call sequences.

\subsubsection{Statistical Model of Call Sequences.}

We can model API call sequence using a discrete time Markov chain [7]. Note that the Markov chain represents the conditional distribution. The use of conditional distribution, instead of joint distribution, reduces the size of the underlying sample space which, for the present problem, corresponds to removing the redundant information from the joint distribution.

The order of a Markov chain represents the extent to which past states determine the present state, i.e., how many lags should be examined when analyzing higher orders. The correlation analysis is very useful for analyzing the order of a Markov chain process. The rationale behind this argument is that if we take into account the past states, it should reduce the surprise or the uncertainty in the present state [7]. The correlation results highlight the $3^{\text {rd }}$ order dependence in call sequences; therefore, we use a $3^{\text {rd }}$ order Markov chain.

Markov chain used to model the conditional distribution has $k$ states, where $k=|\mathbb{S}|$. The probabilities of transitions between these states are detailed in state transition matrix $\mathcal{T}$. An intuitive method to present $\mathcal{T}$ is to couple consecu- (a) Spatial features

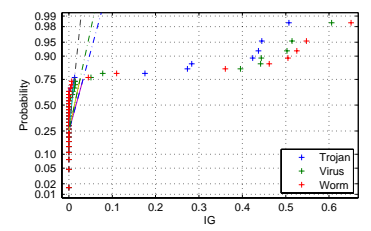

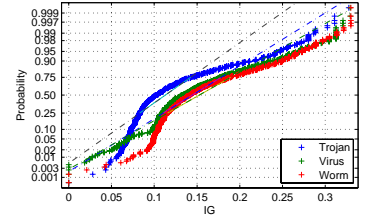

(b) Temporal features
Figure 5: Normal probability distribution plot of Information Gain $(I G)$ for spatial and temporal features

tive states together; as a result, we represent the $3^{\text {rd }}$ order Markov chain in the form of the state transition matrix $\mathcal{T}$.

$$
\mathcal{T}=\left[\begin{array}{cccc}
t_{(0,0),(0,0)} & t_{(0,0),(0,1)} & \ldots & t_{(0,0),(k, k)} \\
t_{(0,1),(0,0)} & t_{(0,1),(0,1)} & \ldots & t_{(0,1),(k, k)} \\
\vdots & \vdots & \ddots & \vdots \\
t_{(0, k),(0,0)} & t_{(0, k),(0,1)} & \ldots & t_{(0, k),(k, k)} \\
t_{(1, k),(0,0)} & t_{(1, k),(0,1)} & \ldots & t_{(1, k),(k, k)} \\
\vdots & \vdots & \ddots & \vdots \\
t_{(k, k),(0,0)} & t_{(k, k),(0,1)} & \ldots & t_{(k, k),(k, k)}
\end{array}\right]
$$

In the following text, we explore the possibility of using probabilities in transition matrix $\mathcal{T}$ to quantify temporal features of API calls.

\subsubsection{Quantification.}

We consider each transition probability a potential feature. However, in the transition matrix we have $k^{n}$ distinct transition probabilities. To select the most discriminative features, we use a feature selection procedure. To this end, we populate two training Markov chains each from a few benign and malware traces. Several information-theoretic measures are proposed in the literature to evaluate the discriminative quality of attributes, such as information gain $(I G \in[0,1])$. Information gain measures the reduction in uncertainty if the values of an attribute are known [7]. For a given attribute $X$ and a class attribute $Y \in\{$ Benign, Malware $\}$, the uncertainty is given by their respective entropies $H(X)$ and $H(Y)$. Then the information gain of $X$ with respect to $Y$ is given by $I G(Y ; X)$ :

$$
I G(Y ; X)=H(Y)-H(Y \mid X)
$$

We compute information gain for each element of $\mathcal{T}$. For a given element, with $a$ counts in the benign training matrix and $b$ counts in the malware training matrix, $I G$ can be computed by [10]:

$I G(a, b)=-\left(\frac{a}{a+b}\right) \cdot \log _{2}\left(\frac{a}{a+b}\right)-\left(\frac{b}{a+b}\right) \cdot \log _{2}\left(\frac{b}{a+b}\right)$

Finally, all potential features are sorted based on their respective $I G$ values to an ordered set $\mathcal{T}_{I G}$. We select $\mathcal{T}_{I G_{\Gamma}} \subset$ $\mathcal{T}_{I G}$ as the final boolean feature set containing $\Gamma$ transitions with top values of $I G$. For this study we have used $\Gamma=500$. 
Table 3: Statistics of executable files used in this study

\begin{tabular}{|c|c|c|c|c|}
\hline $\begin{array}{c}\text { Executable } \\
\text { (Type) }\end{array}$ & Quantity & $\begin{array}{c}\text { Avg. } \\
\text { File Size } \\
(\mathrm{KB})\end{array}$ & $\begin{array}{c}\text { Min. } \\
\text { File Size } \\
(\mathrm{KB})\end{array}$ & $\begin{array}{c}\text { Max. } \\
\text { File Size } \\
(\mathrm{KB})\end{array}$ \\
\hline \hline Benign & 100 & 1,263 & 4 & 104,588 \\
\hline Trojan & 117 & 270 & 1 & 9,277 \\
\hline Virus & 165 & 234 & 4 & 5,832 \\
\hline Worm & 134 & 176 & 3 & 1,301 \\
\hline Total & 516 & 50 & 2 & 1,332 \\
\hline
\end{tabular}

\subsection{Discussion}

We have discussed the formal foundation of our spatiotemporal features' set extracted from the traces of API calls. To analyze the classification potential of these features, we use definition of information gain $(I G)$. The values of $I G$ approaching one represent features with a higher classification potential and vice-versa.

Figure 5 shows the normal probability plot of $I G$ of spatial and temporal features for trojans, viruses and worms. It is interesting to see in Figure 5 the 'skewed' nature of the $I G$ distribution of spatial features. The majority of spatial features have very low values of $I G$, but some of them also have $I G$ values as high as 0.7 . The high $I G$ features can prove valuable in achieving high accuracy. In comparison, the $I G$ distribution of temporal features is fairly regular with most of the $I G$ values lie in the range of $[0.0,0.4]$. Another important observation is that the means of $I G$ distribution of spatial features are $0.10,0.09$ and 0.08 for worms, viruses and trojans respectively. Similarly, the means of $I G$ distribution of temporal features are $0.16,0.14$ and 0.12 for worms, viruses and trojans respectively. Intuitively speaking - on the basis of $I G$ values - we expect that trojans will be most difficult to classify, followed by viruses and worms respectively.

\section{CLASSIFICATION RESULTS AND DIS- CUSSIONS}

In this section, we explain the experiments undertaken to evaluate the classification accuracy of our spatio-temporal malware detection scheme. We have designed two sets of experiments to evaluate the performance of different components of our technique in a systematic manner. In the first set of experiments (Experiment-1), we investigate the merit of using spatio-temporal features' set over standalone spatial or temporal features' set. We take the accuracy from (Experiment-1) as a benchmark and then in the second set of experiments (Experiment-2) we carry out a scalability analysis to identify a minimal subset of API categories that has the potential to deliver the same accuracy. In both sets of experiments, we use the optimal configurations of different classification algorithms ${ }^{2}$ to achieve the best ROC (Receiver Operating Characteristics) curve for them. We first provide a brief description of our dataset and then discuss the accuracy results.

\footnotetext{
${ }^{2}$ In this paper, we have used instance based learner (IB $k$ ), decision tree (J48), Naïve Bayes (NB), inductive rule learner (RIPPER), and support vector machine (SMO) machine learning classification algorithms [18].
}

Table 4: Detection accuracy for spatial (S), temporal $(T)$ and combined (S\&T) features

\begin{tabular}{|c|c|c|c|c|c|c|}
\hline Alg. & IB $k$ & J48 & NB & RIPPER & SMO & Avg. \\
\hline \multicolumn{7}{|c|}{ Trojan } \\
\hline$\overline{\mathrm{S}}$ & 0.926 & 0.910 & 0.777 & $\overline{0.871}$ & $\begin{array}{c}0.760 \\
\end{array}$ & 0.849 \\
\hline $\mathbf{T}$ & 0.958 & 0.903 & 0.945 & 0.932 & 0.970 & 0.942 \\
\hline S\&T & 0.963 & 0.908 & 0.966 & 0.959 & 0.970 & 0.953 \\
\hline \multicolumn{7}{|c|}{ Virus } \\
\hline$\overline{\mathbf{S}}$ & $\overline{c 0.915}$ & $\overline{0.940}$ & $\overline{c 0.859}$ & 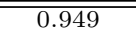 & 0.915 & 0.916 \\
\hline $\mathbf{T}$ & 0.937 & 0.956 & 0.974 & 0.945 & 0.971 & 0.957 \\
\hline S\&T & 0.954 & 0.963 & 0.990 & 0.954 & 0.968 & 0.966 \\
\hline \multicolumn{7}{|c|}{ Worm } \\
\hline$\overline{\mathrm{S}}$ & $\overline{0.973}$ & "0.961 & (20.946 & (0.963 & $\begin{array}{l}0.806 \\
\end{array}$ & (0.930 \\
\hline $\mathbf{T}$ & 0.942 & 0.968 & 0.962 & 0.953 & 0.963 & 0.958 \\
\hline S\&T & 0.958 & 0.966 & 0.984 & 0.975 & 0.963 & 0.969 \\
\hline Avg. & 0.963 & 0.947 & 0.980 & $\overline{0.963}$ & 0.968 & 0.963 \\
\hline
\end{tabular}

\subsection{Dataset}

The dataset used in this study consists of 416 malware and 100 benign executables. All of them are in Win32 portable executable format. The benign executables are obtained from a freshly installed copy of Windows XP and application installers. The malware executables are obtained from a publicly available database called 'VX Heavens Virus Collection' [3]. Table 3 provides the basic statistics of the executables used in our study. These statistics show the diversity of the executables in terms of their file sizes. We have logged the API call traces of these files by executing them on a freshly installed Windows XP. The logging process is carried out using a commercial API call tracer [1]. The API call logs obtained for this study are publicly available at http://www . nexginrc.org/.

\subsection{Experimental Setup}

We now explain the experimental setup used in our study. We have combined the benign executable trace with each type of the malware to create three separate datasets benign-trojan, benign-virus and benign-worm. A stratified 10 -fold cross validation procedure is followed for all experiments reported later in this section. In this procedure, we partition each dataset into 10 folds where 9 of them are used for training and the left over fold is used for testing. This process is repeated for all folds and the reported results are an average of all folds.

For two-class problems, such as malware detection, the classification decision of an algorithm may fall into one of the four categories: (1) True Positive (TP) - correct classification of a malicious executable as malicious, (2) True Negative $(\mathrm{TN})$ - correct classification of a benign executable as benign, (3) False Positive (FP) - wrong classification of a benign executable as malicious, and (4) False Negative $(\mathrm{FN})$ - wrong classification of a malicious executable as benign.

We have carried out the standard ROC analysis to evaluate the accuracy of our system. ROC curves are extensively used in machine learning and data mining to depict the tradeoff between the true positive rate and the false positive rate of a given classifier. We quantify the accuracy (or detection accuracy) of each algorithm by using the area under ROC curve $(0 \leq \mathrm{AUC} \leq 1)$. The high values of AUCs reflect high $t p$ rate $\left(=\frac{T P}{T P+F N}\right)$ and low $f p$ rate $\left(=\frac{F P}{F P+T N}\right)$ [8]. At $\mathrm{AUC}=1, t p$ rate $=1$ and $f p$ rate $=0$. 
Table 5: Detection accuracy results with different feature sets (spatio-temporal) for all malware types used in this study.

\begin{tabular}{|c||c|c|c|c|c||c|}
\hline Alg. & IB $k$ & J48 & NB & RIPPER & SMO & Avg. \\
\hline \hline $\mathbb{S}_{\text {sock }}$ & 0.524 & 0.479 & 0.513 & 0.520 & 0.513 & 0.510 \\
\hline $\mathbb{S}_{m m}$ & 0.966 & 0.946 & 0.952 & 0.942 & 0.926 & 0.946 \\
\hline $\mathbb{S}_{\text {proc }}$ & 0.901 & 0.912 & 0.944 & 0.934 & 0.938 & 0.926 \\
\hline $\mathbb{S}_{\text {io }}$ & 0.809 & 0.782 & 0.841 & 0.823 & 0.839 & 0.819 \\
\hline $\mathbb{S}_{\text {dll }}$ & 0.804 & 0.713 & 0.790 & 0.724 & 0.743 & 0.755 \\
\hline $\mathbb{S}_{\text {reg }}$ & 0.962 & 0.922 & 0.923 & 0.929 & 0.937 & 0.935 \\
\hline $\mathbb{S}_{\text {rm }}$ & 0.500 & 0.480 & 0.501 & 0.499 & 0.521 & 0.500 \\
\hline $\mathbb{S}_{m m} \bigcup \mathbb{S}_{\text {io }}$ & 0.975 & 0.950 & 0.986 & 0.956 & 0.963 & $\mathbf{0 . 9 6 6}$ \\
\hline $\mathbb{S}_{m m} \bigcup \mathbb{S}_{\text {reg }}$ & 0.962 & 0.951 & 0.950 & 0.953 & 0.926 & 0.948 \\
\hline $\mathbb{S}_{\text {io }} \bigcup \mathbb{S}_{\text {reg }}$ & 0.810 & 0.773 & 0.867 & 0.798 & 0.840 & 0.818 \\
\hline $\mathbb{S}_{\text {proc }} \bigcup \mathbb{S}_{\text {io }}$ & 0.973 & 0.924 & 0.988 & 0.941 & 0.961 & 0.957 \\
\hline $\mathbb{S}_{\text {proc }} \bigcup \mathbb{S}_{\text {reg }}$ & 0.905 & 0.919 & 0.946 & 0.916 & 0.937 & 0.924 \\
\hline $\mathbb{S}_{m m} \bigcup \mathbb{S}_{\text {proc }}$ & 0.939 & 0.948 & 0.953 & 0.951 & 0.956 & 0.949 \\
\hline
\end{tabular}

\subsection{Experiment-1}

In the first set of experiments, we evaluate the accuracy of spatial and temporal features separately as well as their combination for detecting trojans, viruses and worms. The detection accuracy results from Experiment-1 are tabulated in Table 4 . The bold values highlight the best accuracy results for a particular classifier and the malware type.

It is interesting to note in Table 4 that spatial and temporal features alone can provide on the average detection accuracies of approximately 0.898 and 0.952 respectively. However, the combined spatio-temporal features' set provide an average detection accuracy of 0.963 - approximately $5 \%$ improvement over standalone features' set.

It is interesting to see that the difference in the relative detection accuracies of all classification algorithms is on the average $4-5 \%$. The last row of Table 4 provides the average of best results obtained for all malware types with each classification algorithm. NB with spatio-temporal features' set provides the best detection accuracy of 0.980 . It is closely followed by SMO which provides the detection accuracy of 0.968. On the other hand, J48 provides the lowest accuracy of 0.947. Another important observation is that once we use the combined spatio-temporal features, the classification accuracies of NB for trojan and virus categories significantly improves to 0.966 and 0.990 respectively.

An analysis of Table 4 highlights the relative difficulty of detecting trojans, viruses and worms. The lowest detection accuracy is obtained for trojans because trojans are inherently designed to appear similar to benign programs. Worms are the easiest to detect while viruses stand in between. Note that this empirical outcome is consistent with our prediction in Section 4.4 based on the $I G$ values of the extracted features for these malware types.

We also show ROC plots in Figure 6 for detection accuracy of different types of malware for all classification algorithms by using spatio-temporal features. The plots further validate that the $t p$ rate of $\mathrm{NB}$ quickly converges to its highest values. The ROC plots also confirm that trojans are the most difficult to detect while it is relatively easier to detect worms.

\subsection{Experiment-2}

The processing and memory overheads of monitoring runtime API calls and storing their traces might lead to sig- nificant performance bottlenecks. Therefore, we have also performed a scalability analysis to quantify the contribution of different functional categories of API calls towards the detection accuracy. By using this analysis, we can identify redundant API categories; as a result, we can find the minimal subset of API categories that provide the same detection accuracy as in Experiment-1. This analysis can help in improving the efficiency and performance of our proposed scheme.

We have performed this analysis in a systematic manner. First we evaluate the detection accuracy of individual API categories namely $\mathbb{S}_{\text {sock }}, \mathbb{S}_{m m}, \mathbb{S}_{\text {proc }}, \mathbb{S}_{i o}, \mathbb{S}_{d l l}, \mathbb{S}_{r e g}$, and $\mathbb{S}_{n m}$. The results of our scalability experiments are tabulated in Table 5. We can conclude from Table 5 that the detection accuracies of $\mathbb{S}_{m m}, \mathbb{S}_{r e g}, \mathbb{S}_{\text {proc }}$, and $\mathbb{S}_{i o}$ is very promising. We have tried all possible combinations $\left({ }^{4} C_{2}=6\right)$ of the promising functional categories. The results of our experiments show that $\mathbb{S}_{m m} \cup \mathbb{S}_{i o}$ provides the best detection accuracy of 0.966. We have also tried higher-order combinations of API categories but the results do not improve significantly.

\section{CONCLUSION AND FUTURE WORK}

In this paper, we have presented our run-time malware analysis and detection scheme that leverages spatio-temporal information available in API calls. We have proven our thesis that combined spatio-temporal features' set increases the detection accuracy than standalone spatial or temporal features' set. Moreover, our scalability analysis shows that our system achieves the detection accuracy of 0.97 by only monitoring API calls from memory management and file I/O categories.

It is important to emphasize that spatial and temporal features use completely different data models; therefore, intuitively speaking they have the potential to provide an extra layer of robustness to evasion attempts. For example, if a crafty attacker manipulates the sequence of system calls to evade temporal features, then spatial features will help to sustain high detection accuracy and vice-versa. In future, we want to quantify the effect of evasion attempts by a crafty attacker on our scheme.

\section{Acknowledgments}

The research presented in this paper is supported by the grant \# ICTRDF/AN/2007/37, for the project titled AISGPIDS, by the National ICT R\&D Fund, Ministry of Information Technology, Government of Pakistan. The information, data, comments, and views detailed herein may not necessarily reflect the endorsements of views of the National ICT R\&D Fund.

\section{REFERENCES}

[1] API Monitor - Spy and display API calls made by Win32 applications, available at http://www. apimonitor.com.

[2] Overview of the Windows API, available at http://msdn.microsoft.com/en-us/library/ aa383723 (VS. 85). aspx.

[3] VX Heavens Virus Collection, VX Heavens, http://vx.netlux.org/.

[4] F. Ahmed, H. Hameed, M.Z. Shafiq, M. Farooq, "Using Spatio-Temporal Information in API Calls with 


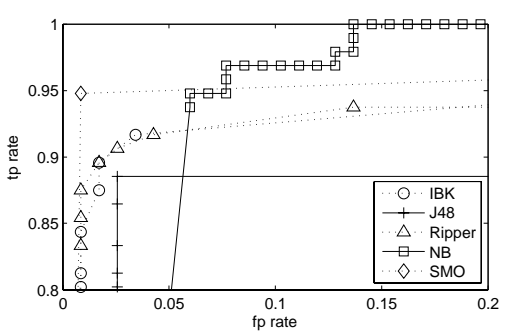

(a) Trojan-Benign (Zoomed)

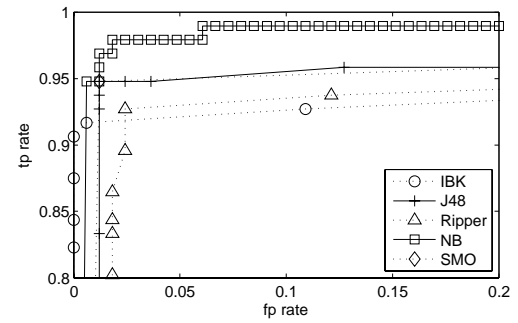

(b) Virus-Benign (Zoomed)

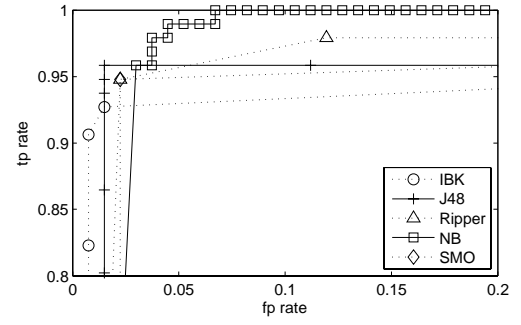

(c) Worm-Benign (Zoomed)

Figure 6: ROC plots for detecting malicious executables using spatial and temporal features.

Machine Learning Algorithms for Malware Detection and Analysis", Technical Report, TR-nexGINRC-2009-42, 2009, available at http://www.nexginrc.org/papers/tr42-faraz.pdf

[5] P. Beaucamps J.-Y. Marion, "Optimized control flow graph construction for malware detection", International Workshop on the Theory of Computer Viruses (TCV), France, 2008.

[6] M. Christodorescu, S. Jha, S.A. Seshia, D. Song, R.E. Bryant, "Semantics-Aware Malware Detection", IEEE Symposium on Security and Privacy (S\&P), IEEE Press, USA, 2005.

[7] T.M. Cover, J.A. Thomas, "Elements of Information Theory", Wiley-Interscience, 1991.

[8] T. Fawcett, "ROC Graphs: Notes and Practical Considerations for Researchers", Techincal Report, HP Labs, CA, 2003-4, USA, 2003.

[9] S. Forrest, S.A. Hofmeyr, A. Somayaji, T.A. Longstaff, "A Sense of Self for Unix Processes", IEEE Symposium on Security and Privacy (S\&P), IEEE Press, pp. 120-128, USA, 1996

[10] J. Han, M. Kamber, "Data Mining: Concepts and Techniques", Morgan Kaufmann, 2000.

[11] D. Mutz, F. Valeur, C. Kruegel, G. Vigna, "Anomalous System Call Detection", ACM Transactions on Information and System Security (TISSEC), 9(1), pp. 61-93, ACM Press, 2006.

[12] C. Parampalli, R. Sekar, R. Johnson, "A Practical
Mimicry Attack Against Powerful System-Call Monitors", ACM Symposium on Information, Computer and Communications Security (AsiaCCS), pp. 156-167, Japan, 2008.

[13] M.Z. Shafiq, S.A. Khayam, M. Farooq, "Embedded Malware Detection using Markov n-grams", Detection of Intrusions and Malware, and Vulnerability Assessment (DIMVA), pp. 88-107, Springer, France, 2008.

[14] G. Tandon, P. Chan, "Learning Rules from System Call Arguments and Sequences for Anomaly Detection", ICDM Workshop on Data Mining for Computer Security (DMSEC), pp. 20-29, IEEE Press, USA, 2003.

[15] D. Wagner, P. Soto, "Mimicry Attacks on Host-Based Intrusion Detection Systems", ACM Conference on Computer and Communications Security (CCS), pp. 255-264, ACM Press, USA, 2002.

[16] C. Warrender, S. Forrest, B. Pearlmutter, "Detecting Intrusions Using System Calls: Alternative Data Models", IEEE Symposium on Security and Privacy (S\&P), pp. 133-145, IEEE Press, USA, 1999.

[17] A. Wespi, M. Dacier, H. Debar, "Intrusion Detection Using Variable-Length Audit Trail Patterns", Recent Advances in Intrusion Detection (RAID), pp. 110-129, Springer, France, 2000.

[18] I.H. Witten, E. Frank, "Data mining: Practical machine learning tools and techniques", Morgan Kaufmann, 2nd edition, USA, 2005. 